\title{
STELLAR POPULATIONS IN BULGES OF SPIRAL GALAXIES
}

\author{
PASCALE JABLONKA \\ D.A.E.C., UMR 8631, Place Jules Janssen, 92195 Meudon, France \\ JAVIER GORGAS \\ Dpt. de Astrofisica, Universidad Complutense, Madrid, Spain \\ PAUL GOUDFROOIJ \\ Space Telescope Science Institute, Baltimore, USA
}

\begin{abstract}
We present a few results of a work in progress tackling the radial spectroscopic properties of bulges of spiral galaxies.
\end{abstract}

Keywords: spiral galaxies, evolution of galaxies, stellar population

\section{Introduction}

We have conducted a spectroscopic analysis of the bulges of a sample of 31 spiral galaxies, spanning the Hubble sequence from S0 to Scd type, in order to study the radial distribution of their stellar population properties. This is the first sample of this size, allowing at last some statistics. We have in mind the following considerations:

- Radial spectral information brings insights on the structure of the systems which are considered, as it unveils the light/mass spatial distribution. It also gives important clues on time scales at play: either directly from age indicators, or, particularly for old populations, by considering abundance ratios of chemical elements appearing with the explosion of different supernova types, like type Ia and type II.

- Moreover, debates on the formation and evolution of galaxies are, specially for nearby galaxies, more than often based on observations of central indices, or, more precisely, integrated indices in apertures centered on the galaxy nuclei and of small sizes when compared to the size of the galaxies. Meanwhile, models generally follow/predict the global properties of the galaxies, which would find a more natural echo in mean observational quantities. This is also the case of observations at high redshift. Tracking the spatial distribution of the stellar population allows one to trace back these galactic mean spectral indices.

- Numerical simulations nowadays offer a good deal of various proposals for the origin of the formation of bulges, however data are crucially missing to tackle crucial questions like: how far can we push the parallel between ellipt- 


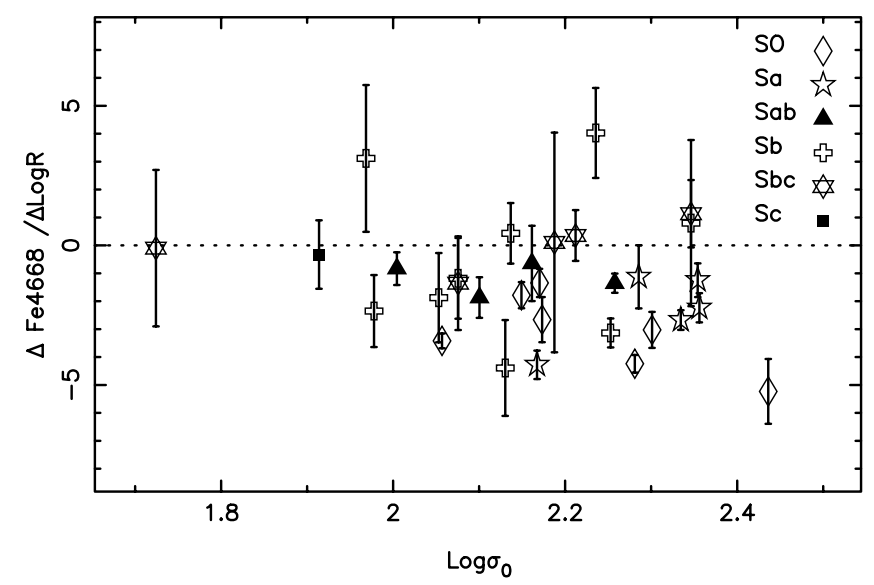

Figure 1. Radial gradient of the Fe4668 index as a function of the bulge central velocity dispersion.

icals and bulges? By how much do the discs and bulges interplay and when in the galaxy history?

\section{Observations and Results}

We have selected purely edge-on spirals in order to free our spectra from any disc light. We also centered the slit of the spectrograph and placed it perpendicular to the plane of the discs to avoid any trend due to the rotation of the bulges. We finally could measure our indices to at least one bulge effective radius $\left(r_{\text {eff }}\right)$ and up to $5 r_{\text {eff }}$.

We chose to measure our spectral features in the Lick system (Worthey et al., 1994), for purposes of comparison with large samples of ellipticals. Our interest for the present time is not to give absolute values in age or metallicity $(\mathrm{Z})$, but to understand whether variations of our indices are due to a radial change in age, in metallicity or in both.

As there is no one-to-one relation between any individual Lick index and age or even the abundance of one single chemical element (Worthey, 1994), we use as many indices as possible and consider their different relative sensitivities to age and metallicity. The wavelength coverage of our spectra enable us to measure 25 indices, from $\mathrm{H} \delta$ to $\mathrm{TiO}$.

We present here the example of Fe4668 and $\mathrm{H} \gamma_{A}$. Fe4668 is around 5 times more sensitive to metallicity than to the logarithm of the age, while $\mathrm{H} \gamma_{A}$ is about as sensitive to $\log (\mathrm{age})$ as to metallicity. Figure 1 and Figure 2 present the radial gradients of these two indices as a function of the bulge central velocity dispersion. A different symbol is used for each Hubble type. The error bars of our measurements are shown, they are the result of a detailed error propagation from the 


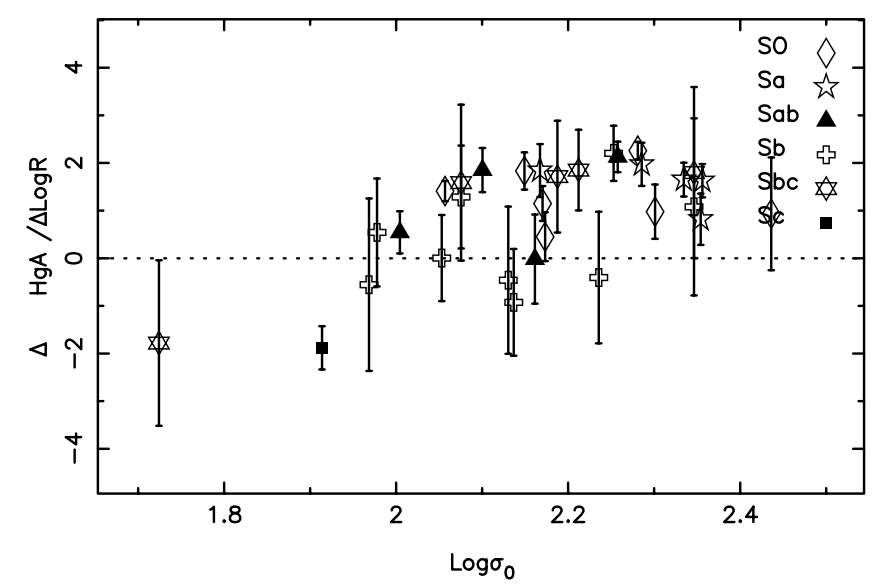

Figure 2. Radial gradient of the $\mathrm{H} \gamma_{A}$ index as a function of the bulge central velocity dispersion.

first steps of data reduction to the final measurements. We show as dotted line the position of a gradient slope of 0 , which would correspond to the absence of any radial variation of the index.

The distribution of $\Delta \mathrm{Fe} 4668 / \Delta \log r$ is flat with a mean value of -2.5 and a r.m.s. dispersion of 1.4. The distribution of $\Delta \mathrm{H}_{\gamma_{A}} / \Delta \log r$ is flat with a mean value of 1.5 and a dispersion of 0.8 .

Two remarks can be made: (Note: We discuss the mean observed trends and do not address any individual galaxy that might deviate slightly from those trends.)

- While Fe4668 decreases, $\mathrm{H} \gamma_{A}$ increases from the center to the outer edges of the bulges. The clear radial change of Fe4668, both by itself and as compared to the variation of $\mathrm{H} \gamma_{A}$, on one hand, the sign of the slope of the gradients, on the other hand, support the view of a variation in metallicity as primary underlying factor, the bulge inner parts being more metal-rich than the outer regions. This is: a pure age gradient would make Fe4668 slope about ten times smaller than that observed, and $\Delta \mathrm{H}_{\gamma_{A}} / \Delta \log r$ greater than $\Delta \mathrm{Fe} 4668 / \Delta \log r$, therefore this hypothesis is discarded.

- Neither $\mathrm{H} \gamma_{A}$ nor Fe4668 radial gradients show any dependence on the Hubble type of the galaxies. This is also the case for the other indices. The loose connection between the morphological classification of the spirals and the bulge stellar population had been previously noticed for the central indices of bulges (Jablonka, Arimoto and Martin, 1996). But while the bulge central indices are found to be related to the bulge central velocity dispersion, there is no comparable trend for the radial gradients (except for $\mathrm{Mg}_{2}$ ).

As to the comparison between bulges and ellipticals, interestingly, the relation between gradients in ellipticals and their central velocity dispersion is a longstanding debate (Gonzalez and Gorgas 1996; Kobayashi and Arimoto 1999). A further 
comparison with the results quoted in Gorgas et al. (1997) reveals that the mean slope of the $\mathrm{Mg}_{2}$ gradient in ellipticals coincides with the values we find for the bulges of our sample $(-0.055 \pm 0.025$ vs $-0.047 \pm 0.023)$

\section{Conclusion}

While by no means have we exhausted all the information of our dataset yet, we already see quite clearly that any scenario of formation of bulges requiring long time scales would be in contradiction with the observations. We also stress the apparent continuity between elliptical and bulge properties.

\section{References}

Gonzalez, J.J. and Gorgas, J.: 1996, Fresh views of elliptical galaxies. ASPC Series 86, p. 225.

Gorgas, J., Pedraz, S., Guzman, R., Cardiel, N. and Gonzalez J.J.: 1997, ApJ 481, L19.

Jablonka, P., Martin, P. and Arimoto, N.: 1996, AJ 112, 1415.

Kobayashi, C. and Arimoto, N.: 1999, AJ 257, 573.

Worthey, G.: 1994, ApJS 95, 107.

Worthey, G., Faber, S.M., Gonzalez, J.J. and Burstein, D.: 1994, APJS 94, 687. 